\title{
Better Refined Adsorption Isotherm than BET Equation
}

\author{
Daekyoum Kim \\ Kwongmyoung-si Neobudae-ro, 32 Beon-kil, South Korea \\ Email: dkkim5019@naver.com
}

Received 9 March 2016; accepted 3 May 2016; published 6 May 2016

Copyright (C) 2016 by author and Scientific Research Publishing Inc.

This work is licensed under the Creative Commons Attribution International License (CC BY). http://creativecommons.org/licenses/by/4.0/

c) (i) Open Access

\begin{abstract}
During studying the heat capacity of metals and brightening more than the original Lena's image, the temperature increasing term obtained in binomial expansion is transformed into the adsorption increasing term and thereafter we have derived the total adsorption rate equation with it. In the first layer the quantization does not occur and from $2^{\text {nd }}$ layer to $\mathbf{n}^{\text {th }}$ layer the quantization occurs. So as to get the total adsorption rate equation we add the quantized terms of the second to $\mathrm{n}^{\text {th }}$ layers to the non-quantized term of the first layer. All terms are based on the unit surface sites. Instead of the unit surface sites, the new adsorption site term appears in the denominator of the adsorption equation. Hence the adsorption equations come out much better than BET equation. The surface area is also calculated through the integration of the adsorption isotherm equation excluding the first layer adsorption equation from the inflection point to the wanted relative pressure.
\end{abstract}

\section{Keywords}

Refined BET, Binomial, Transforming, Adsorption Increasing Term, Surface Area, Inflection Point

\section{Introduction}

We derived the heat capacity equations of metals and then used consistent step multiplication of the appropriate binomial equations [1]. They are fitted to experimental data well [2]. The heat capacity equation (type V) and the adsorption equation (type II) draw sigmoid (S character) lines all together. And they are symmetrical with each other. The measurement gases of heat capacity are hydrogen and helium. The adsorption gases are vapor and nitrogen. The movements of their measurement gases are different. The formers are expansion and the later contraction.

The most important term in the derivation of heat capacity equation was the temperature increasing term, 
$g_{b} \frac{N_{n}}{N_{n-1}-N_{n}}$. In case of adsorption it becomes $g_{a} \frac{N_{n}}{N_{n-1}-N_{n}}$. Here $N_{n}$ and $N_{n}$ are the total molecules adsorbed at the layers of $n$ and $n-1$. Let us put $g_{a}$ which is unit in data fitting as the constant. The constant affects the adsorption equation from starting to ending like other constants. If $g_{a} \frac{N_{n}}{N_{n-1}-N_{n}}$ is transformed into $\frac{1}{m}$ power of $z$ in every derivation of adsorption such as $g_{a} \frac{N_{n}}{N_{n-1}-N_{n}}=z^{\frac{1}{m}}$ [3], the more advanced adsorption equation than BET eq. comes out. Hence we get the surface area.

\section{Statistical Modeling of Adsorption Isotherm}

Suppose each layer has one binomial equation. And suppose $N_{1}$ molecules are adsorbed on $B$ localized sites of the unit surface layers of the adsorbent and $\frac{N_{2}}{m}, \frac{N_{3}}{m}, \cdots, \frac{N_{n-1}}{m}, \frac{N_{n}}{m}$ molecules on $\frac{N_{1}}{m}, \frac{N_{2}}{m}, \cdots, \frac{N_{n-2}}{m}, \frac{N_{n-1}}{m}$ sites made of $1 s t$ to $(n-1)$ th layers in sequence. Here $m$ is quantization constant. The first adsorption layer has $D_{l}$ adsorption energy and. the second to $n t h$ layers, $D_{h}$. Hence the adsorption probability on the first layer is $P_{l}=W_{l} \exp \left(-D_{l} / k_{K} t_{a}\right)$ and the non-adsorption probability $1-W_{l} \exp \left(-D_{l} / k_{K} t_{a}\right)$. Here $W_{l}$ is the adsorption constant of the first adsorption layer. $t_{a}$ is similar to $t_{s}$ in heat capacity equation. The adsorption constant, $W_{l}$ is calculable quantity. It is the expression to combine the rotation and vibration energy of the adsorbed molecules in the first layer. $k_{K}$ is similar to $k_{B}$ (Boltzmann's constant) [2]. In the combination calculation of the first layer $N_{1}$ can take from 0 to $B$ as variables in sequence. Then the binomial equation of the first layer becomes [1]

$$
W_{1}\left(B, N_{1}\right)=\left(P_{l}+1-P_{l}\right)^{B}=1 \cong \sum_{N_{1} \leq B}^{B} \frac{B !}{\left(B-N_{1}\right) ! N_{1} !} P_{l}^{N_{1}}\left(1-P_{l}\right)^{B-N_{1}}
$$

Next the adsorption probability of from $N_{2}$ to $N_{n}$ layers is $P_{h}=W_{h} \exp \left(-D_{h} / k_{K} t_{a}\right)$ and the nonadsorption probability $1-W_{h} \exp \left(-D_{h} / k_{K} t_{a}\right)$. The binomial equations for from $N_{2}$ to $N_{n}$ layers are

$$
\begin{aligned}
& W_{2}\left(N_{1}, \frac{N_{2}}{m}\right)=\left(P_{h}+1-P_{h}\right)^{N_{1}}=1 \cong \sum_{\frac{N_{2}}{m} \leq N_{1}}^{N_{1}} \frac{N_{1} !}{\left(N_{1}-\frac{N_{2}}{m}\right) !\left(\frac{N_{2}}{m}\right) !} P_{h}^{\frac{N_{2}}{m}}\left(1-P_{h}\right)^{N_{1}-\frac{N_{2}}{m}} \\
& W_{n-1}\left(\frac{N_{n-2}}{m}, \frac{N_{n-1}}{m}\right)=\left(P_{h}+1-P_{h}\right)^{\frac{N_{n-2}}{m}}=1 \cong \sum_{\frac{N_{n-1}}{m} \leq \frac{N_{n-2}}{m}}^{\frac{N_{n-2}}{m}} \frac{\frac{N_{n-2}}{m} !}{\left(\frac{N_{n-2}}{m}-\frac{N_{n-1}}{m}\right) !\left(\frac{N_{n-1}}{m}\right) !} \times P_{h}^{\frac{N_{n-1}}{m}}\left(1-P_{h}\right)^{\frac{N_{n-2}}{m}-\frac{N_{n-1}}{m}} \\
& W_{n}\left(\frac{N_{n-1}}{m}, \frac{N_{n}}{m}\right)=\left(P_{h}+1-P_{h}\right)^{\frac{N_{n-1}}{m}}=1 \cong \sum_{\frac{N_{n}}{m} \leq \frac{N_{n-1}}{m}}^{\frac{N_{n-1}}{m}} \frac{\frac{N_{n-1}}{m} !}{\left(\frac{N_{n-1}}{m}-\frac{N_{n}}{m}\right) !\left(\frac{N_{n}}{m}\right) !} \times P_{h}^{\frac{N_{n}}{m}}\left(1-P_{h}\right)^{\frac{N_{n-1}}{m}-\frac{N_{n}}{m}}
\end{aligned}
$$

Let us multiply Equation (1) and Equation (2) side by side. Then for

$$
B \geq N_{1} \geq \frac{N_{2}}{m} \geq \cdots \geq \frac{N_{n-1}}{m} \geq \frac{N_{n}}{m}
$$




$$
\begin{aligned}
& W_{a T}\left(B, N_{1}, \frac{N_{2}}{m}, \cdots, \frac{N_{n-1}}{m}, \frac{N_{n}}{m}\right)=W_{1} W_{2} \cdots W_{n-1} W_{n} \cong 1 \\
& \cong \sum_{\frac{N_{n}}{m} \leq \frac{N_{n-1}}{m}}^{\frac{N_{n-1}}{m}} \cdots \sum_{N_{1} \leq B}^{B} \frac{B ! P_{l}^{N_{1}}\left(1-P_{l}\right)^{B-N_{1}} P_{h}^{N-N_{1}}\left(1-P_{h}\right)^{N_{1}-\frac{N_{n}}{m}}}{\left(B-N_{1}\right) !\left(N_{1}-\frac{N_{2}}{m}\right) ! \cdots\left(\frac{N_{n-1}}{m}-\frac{N_{n}}{m}\right) !\left(\frac{N_{n}}{m}\right) !} \\
& =\sum_{\frac{N_{n}}{m} \leq \frac{N_{n-1}}{m}}^{\frac{N_{n-1}}{m}} \cdots \sum_{N_{n} \leq B}^{B} W_{a t}
\end{aligned}
$$

In the above $N=N_{1}+\frac{N_{2}}{m}+\cdots+\frac{N_{n-1}}{m}+\frac{N_{n}}{m}$

In Equation (3) the largest term dominates the equation. So the total differential of Equation (3) becomes the zero which requires that the coefficients of all terms should be zero. Hence by using Stirling's approximation we solve the equation, $W_{a t}$. The first equation (Equation (1)) becomes

$$
\frac{B-N_{1}}{\beta_{a}}\left(g_{a} \frac{N_{n}}{N_{n-1}-N_{n}}\right)=N_{1}-\frac{N_{2}}{m}
$$

In [4] $m$ value should be corrected as those in the present figure (Figure 1). The constants have three parts of $m \succ 1, m=1, m \prec 1$. As we see in Figure 1, bonding constants mean quantization. If $m \succ 1$, bonding occurs in many directions. If $m=1$ no quantization occur and if $m \prec 1$, the adsorptions are interrupted in many directions. In Equation (4)

$$
\begin{gathered}
\beta_{a}=\frac{W_{h}}{W_{l}}\left\{\exp \frac{-\left(D_{l}-D_{h}\right)}{k_{K} t_{a}}\right\}\left\{\frac{1-W_{l} \exp \frac{-D_{l}}{k_{K} t_{a}}}{1-W_{h} \exp \frac{-D_{h}}{k_{K} t_{a}}}\right\} \\
g_{a}=1-W_{h} \exp \left(\frac{-D_{h}}{k_{K} t_{a}}\right)=1-\exp \frac{-D_{h}}{k_{K} t_{a}} \text { for } W_{h}=1
\end{gathered}
$$

It is possible that $g_{a}$ is put as unit.

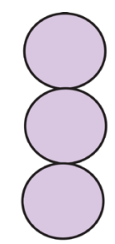

$\mathrm{m}=2$

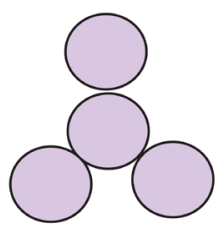

$\mathrm{m}=3$

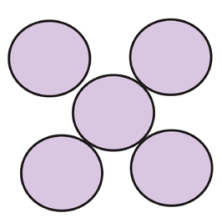

$m=4$

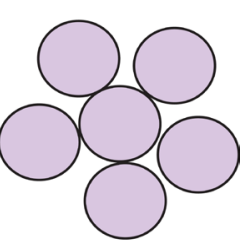

$\mathrm{m}=5$

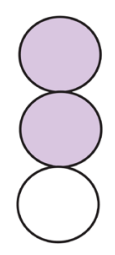

$\mathrm{m}=0.5$

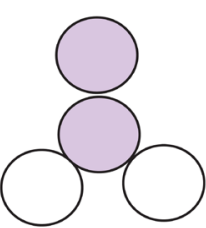

$m=0.333$

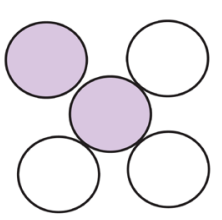

$\mathrm{m}=0.25$

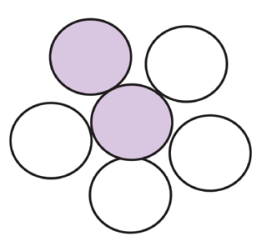

$\mathrm{m}=0.2$

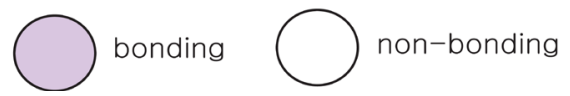

Figure 1. Bonding constant and non-bonding constant in statistic quantization. 
The next equations are

$$
\begin{aligned}
& \left(N_{1}-\frac{N_{2}}{m}\right)\left(g_{a} \frac{N_{n}}{N_{n-1}-N_{n}}\right)=\frac{N_{2}}{m}-\frac{N_{3}}{m} \\
& \vdots \\
& \left(\frac{N_{n-3}}{m}-\frac{N_{n-2}}{m}\right)\left(g_{a} \frac{N_{n}}{N_{n-1}-N_{n}}\right)=\frac{N_{n-2}}{m}-\frac{N_{n-1}}{m} \\
& \left(\frac{N_{n-2}}{m}-\frac{N_{n-1}}{m}\right)\left(g_{a} \frac{N_{n}}{N_{n-1}-N_{n}}\right)=\frac{N_{n-1}}{m}-\frac{N_{n}}{m} \quad n=2,3,4, \cdots
\end{aligned}
$$

In Equations (4) and (5) let us put $g_{a} \frac{N_{n}}{N_{n-1}-N_{n}}=z^{\frac{1}{m}}$, then $z=p / p_{0}$ which is same as Equation (9) of [5] solved by using the chemical potential

$$
\begin{aligned}
& \frac{B-N_{1}}{\beta_{a}} Z^{\frac{1}{m}}=N_{1}-\frac{N_{2}}{m} \\
& \left(N_{1}-\frac{N_{2}}{m}\right) Z^{\frac{1}{m}}=\frac{N_{2}}{m}-\frac{N_{3}}{m} \\
& \vdots \\
& \left(\frac{N_{n-3}}{m}-\frac{N_{n-2}}{m}\right) Z^{\frac{1}{m}}=\frac{N_{n-2}}{m}-\frac{N_{n-1}}{m} \\
& \left(\frac{N_{n-2}}{m}-\frac{N_{n-1}}{m}\right) Z^{\frac{1}{m}}=\frac{N_{n-1}}{m}-\frac{N_{n}}{m}
\end{aligned}
$$

In Equation (6) add side by side and rearrange

$$
\frac{B-N_{1}}{\beta_{a}}\left(\frac{Z^{\frac{1}{m}}-Z^{\frac{n}{m}}}{1-Z^{\frac{1}{m}}}\right)=N_{1}-\frac{N_{n}}{m}
$$

In Equation (6) multiple side by side and rearrange

$$
\frac{B-N_{1}}{\beta_{a}} z^{\frac{n-1}{m}}=\frac{N_{n-1}}{m}-\frac{N_{n}}{m}
$$

We solve Equation (8) with $g_{a} \frac{N_{n}}{N_{n-1}-N_{n}}=z^{\frac{1}{m}}$ in order to eliminate $N_{n-1}$

$$
\frac{B-N_{1}}{\beta_{a}} \frac{1}{g_{a}} z^{\frac{n}{m}}=\frac{N_{n}}{m}
$$

We solve Equation (7) with Equation (9) in order to eliminate $N_{n}$

$$
\frac{N_{1}}{B}=\frac{\frac{z^{\frac{1}{m}}-z^{\frac{n}{m}}}{1-z^{\frac{1}{m}}}+\frac{z^{\frac{n}{m}}}{g_{a}}}{\beta_{a}+\frac{z^{\frac{1}{m}}-z^{\frac{n}{m}}}{1-z^{\frac{1}{m}}}+\frac{z^{\frac{n}{m}}}{g_{a}}}
$$

Equation (10) represents the adsorption amount of the first layer. It is Langmuir equation. The quantization values $(m)$ and the numbers of the adsorption layer $(n)$ are influential on the determination of $N_{1}$. All values 
of the parameters $\left(\beta_{a}, g_{a}, n, m\right)$ directly participate in the determination of $N_{1}$ and following $N$. The value of $g_{a}$ is much influential on the determination of the last term $\left(z^{\frac{n}{m}}\right)$. But it does not much increase or decrease of $N_{1}$ since it exists in the denominator and nominator. At $m=1$ it becomes

$$
\frac{N_{1}}{B}=\frac{\frac{z-z^{n}}{1-z}+\frac{z^{n}}{g_{a}}}{\beta_{a}+\frac{z-z^{n}}{1-z}+\frac{z^{n}}{g_{a}}}
$$

Equation (11) is the same as Equation (10) obtained by using chemical potential in [1]. Therefore the total adsorption amount per unit surface $(B)$, that is, the adsorption isotherm for from first layer to the last $(n)$ layer becomes by using Equations (6) and (10) as follows

$$
\begin{aligned}
& \frac{N}{B}=\frac{N_{1}}{B}+\frac{N_{2}}{m B}+\frac{N_{3}}{m B}+\cdots+\frac{N_{n-1}}{m B}+\frac{N_{n}}{m B} \\
& =\frac{1}{B}\left\{N_{1}+\left(\frac{N_{2}}{m}-\frac{N_{3}}{m}\right)+2\left(\frac{N_{3}}{m}-\frac{N_{4}}{m}\right)+\cdots+(n-2)\left(\frac{N_{n-1}}{m}-\frac{N_{n}}{m}\right)+(n-1) \frac{N_{n}}{m}\right\} \\
& =\frac{a}{\beta_{a}+a}\left\{1+\frac{1}{a}\left(\frac{z^{\frac{2}{m}}-z^{\frac{n}{m}}}{\left(1-z^{\frac{1}{m}}\right)^{2}}+\frac{n-1}{g_{a}} z^{\frac{n}{m}}\right)\right\}
\end{aligned}
$$

In the above

$$
a=\frac{z^{\frac{1}{m}}-z^{\frac{n}{m}}}{1-z^{\frac{1}{m}}}+\frac{z^{\frac{n}{m}}}{g_{a}}
$$

And the total adsorption rate is a linear function of $z$ made of four constants $\left(\beta_{a}, g_{a}, n, m\right)$. The equation draws BET-like lines and fits BET type experimental data well. At $m=1$ it becomes

$$
\frac{N}{B}=\frac{1}{B}\left(N_{1}+N_{2}+N_{3}+\cdots+N_{n-1}+N_{n}\right)=\frac{a^{\prime}}{\beta_{a}+a^{\prime}}\left\{1+\frac{1}{a^{\prime}}\left(\frac{z^{2}-z^{n}}{(1-z)^{2}}+\frac{n-1}{g_{a}} z^{n}\right)\right\}
$$

In the above

$$
a^{\prime}=\frac{z-z^{n}}{1-z}+\frac{z^{n}}{g_{a}}
$$

If the measurement gas is nitrogen, the general empirical formulae, $A_{m}=1.091\left(\frac{M}{\rho N}\right)^{\frac{2}{3}} \times 10^{16}$ is introduced into. $S=\frac{x_{m}}{M} \times N \times A_{m} \times 10^{-20}$ to get the specific surface area of the adsorbate, $S$ [6]. Then $M$ is the molecular weight of nitrogen and $\rho$ density of nitrogen and $N$ Avogadro number, Hence from empirical formulae $A_{m}=\AA^{2} /$ number is used. The monolayer capacity, $x_{m}$ should also be substituted by the value of the integration of Equation (12) subtracted by the surface adsorption isotherm $\left(N_{1} / B\right)$.

\section{Result and Discussion}

The base of Equations ((10) and (12)) is $\beta_{a}+a$. So we may use the word, the rate without considering dimension. It affects the equation totally. Figure 2 shows the total adsorption rate according to the values of $\beta_{a}$. In 
accordance with the values of $z$ approaching units, the total adsorption rates approach closely with one another. This seems to mean that the adsorption heat of the first layer is same as those of $2 \sim n$ layers. We call $m$ the quantization values. It seems to have same notion as the quantization appears in quantum mechanism. We are dealing statistical quantization which should exist in statistics. We can discern them, three cases. $m \succ 1, m=1$ and $m \prec 1$. The cases of $m \succ 1$ and $m=1$ explain bonding and $m \prec 1$ explains the existence of the interruptions for bonding. Figure 3 explains the increase of the adsorption rate according to $m$ values.

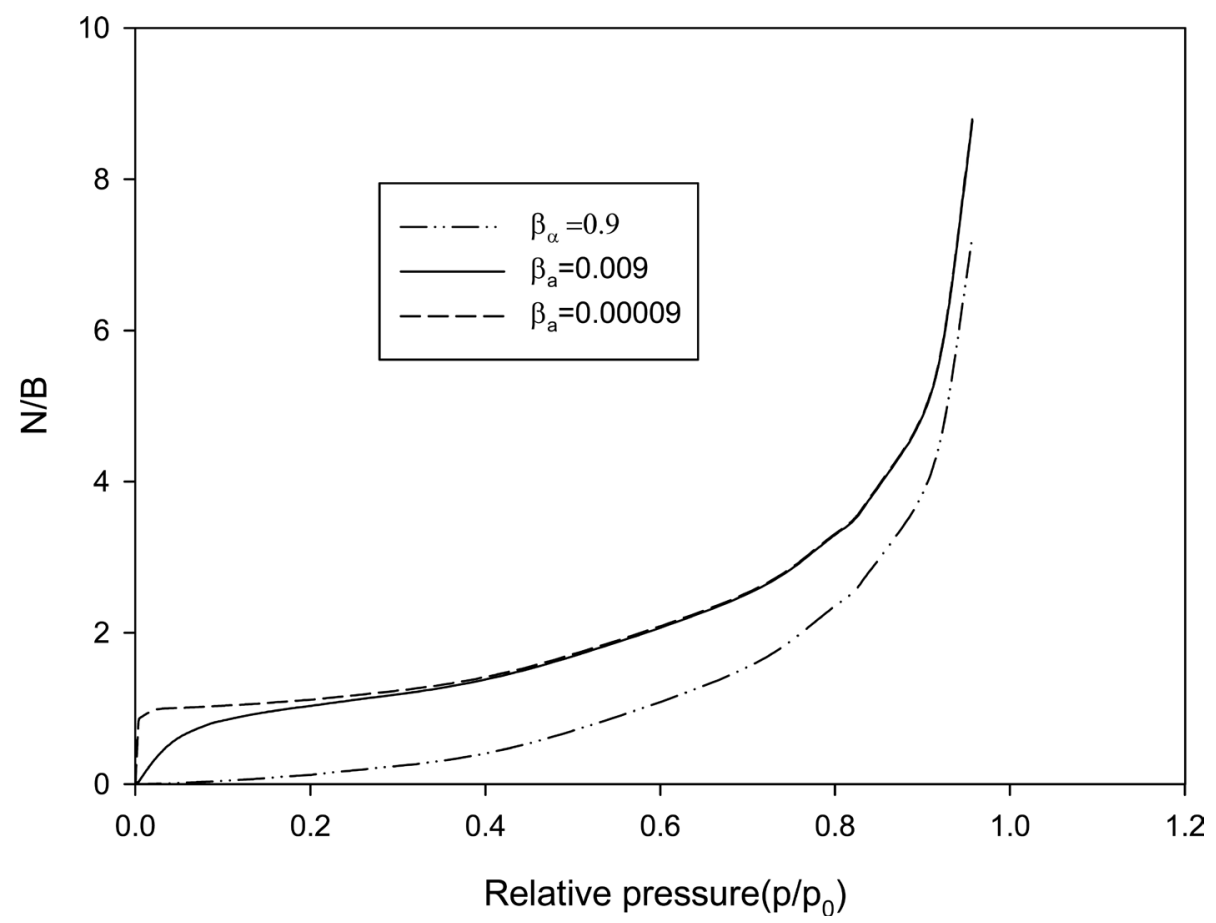

Figure 2. Theoretical adsorption isotherm curve, Equation (12) $\left(\mathrm{m}=0.7, \mathrm{n}=4, \mathrm{~g}_{\mathrm{a}}=0.5\right)$ with respect to $\beta_{\mathrm{a}}$ values.

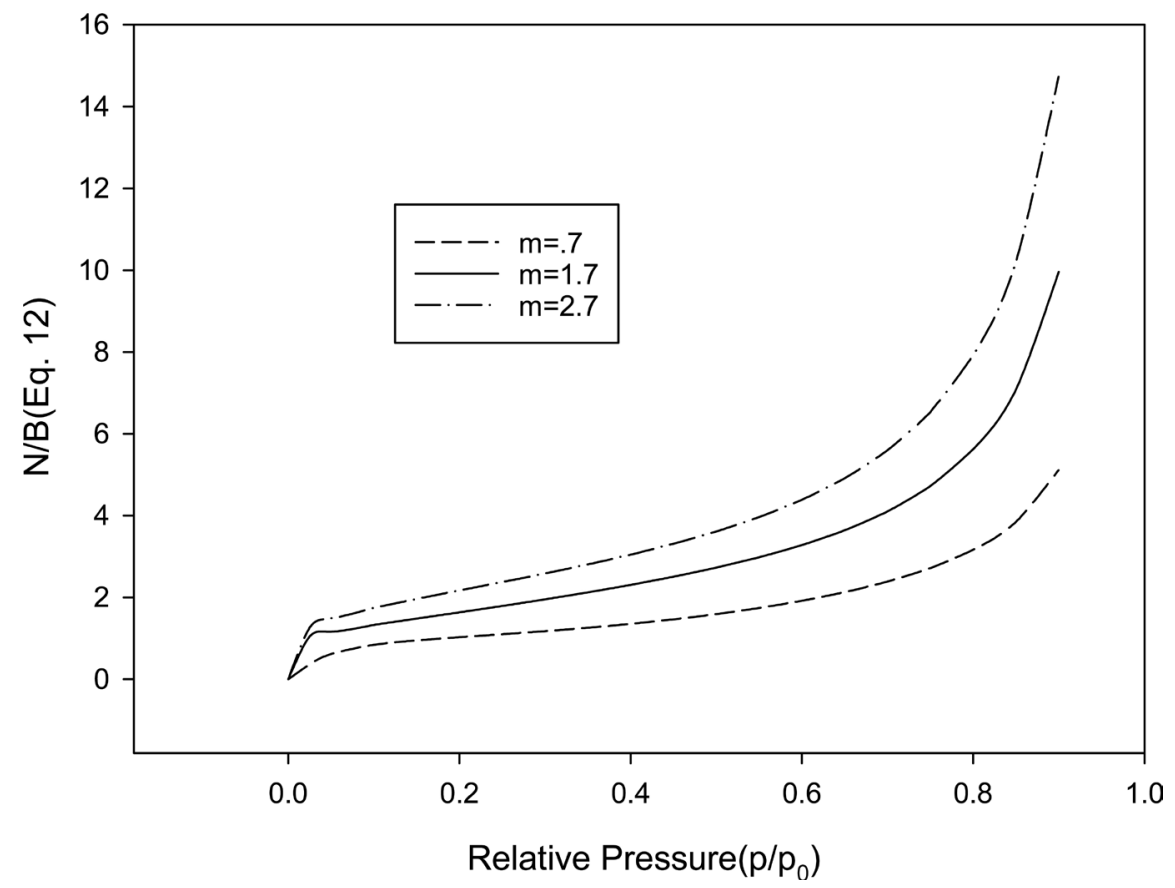

Figure 3. Theoretical adsorption isotherm curve, Equation (12) $\left(n=4, \beta_{a}=0.00901, g_{a}=1.0\right)$ with respect to $m$ values. 
Less $m$ than unit seem to interrupt the bonding. Figure 4 represents the total adsorption rates according to $n$ values. At less $z$ than 0.5 the isotherms show the same adsorption rates. The constant $g_{a}$ showed in Figure 5 should be positive. When $g_{a}=0.5123$ and $g_{a}=0.1122$, the constants draw the type II isotherm, but at $g_{a}=0.0123$ the different type (type IV) of the isotherm appear. Figure 6 shows $N_{1} / B$ with respect to the relative pressure like Langmuir's lines which can't become unit even if $a$ is very large or $\beta_{a}$ much smaller. The parameter values of Figure 6 come from Figure 7 and Figure 8. According to the above variations we optimized two kinds of the experimental adsorption isotherm data showed in Figure 7 [6] and Figure 8 [7] using trial and error method. The experimental data of Figure 7 are obtained from the Figures 2-10 of [6]. The

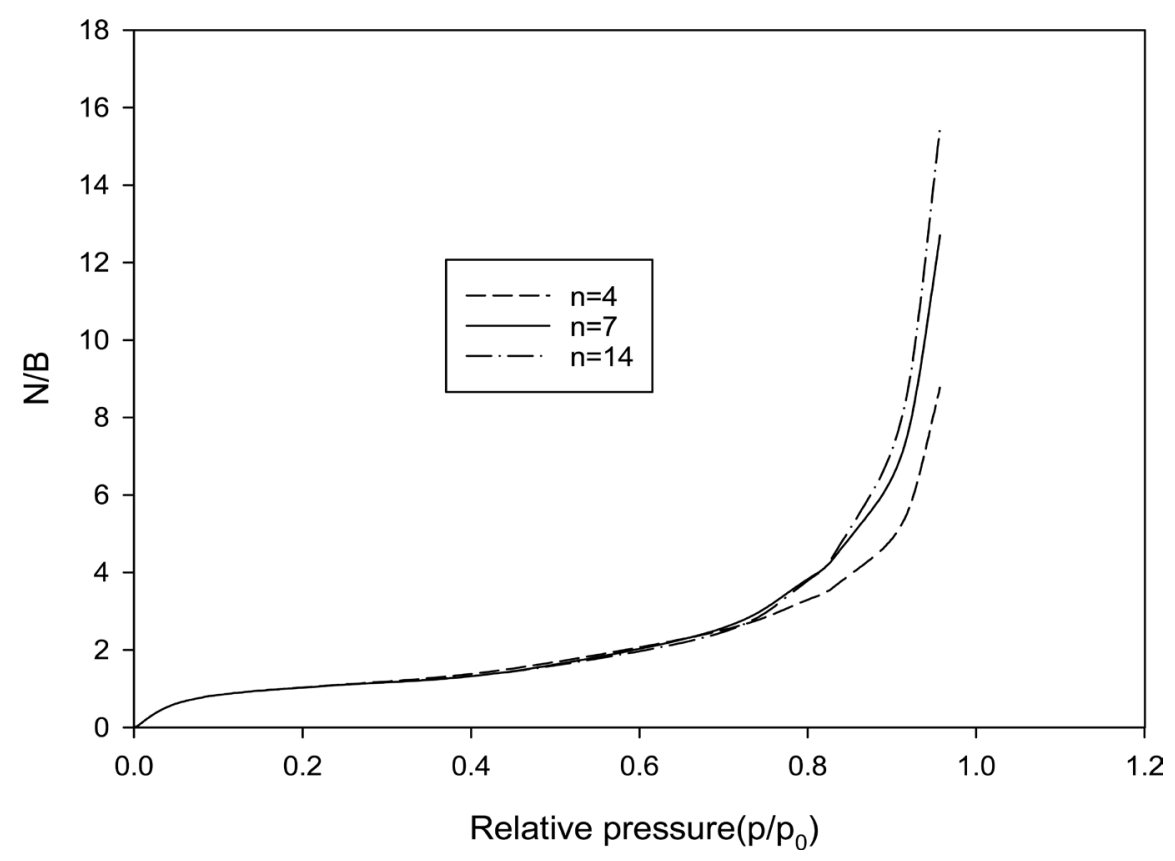

Figure 4. Theoretical adsorption isotherm curves, Equation (12) $\left(\beta_{\mathrm{a}}=0.009, \mathrm{~g}_{\mathrm{a}}=0.5, \mathrm{~m}=0.7\right)$ with respect to $\mathrm{n}$ values.

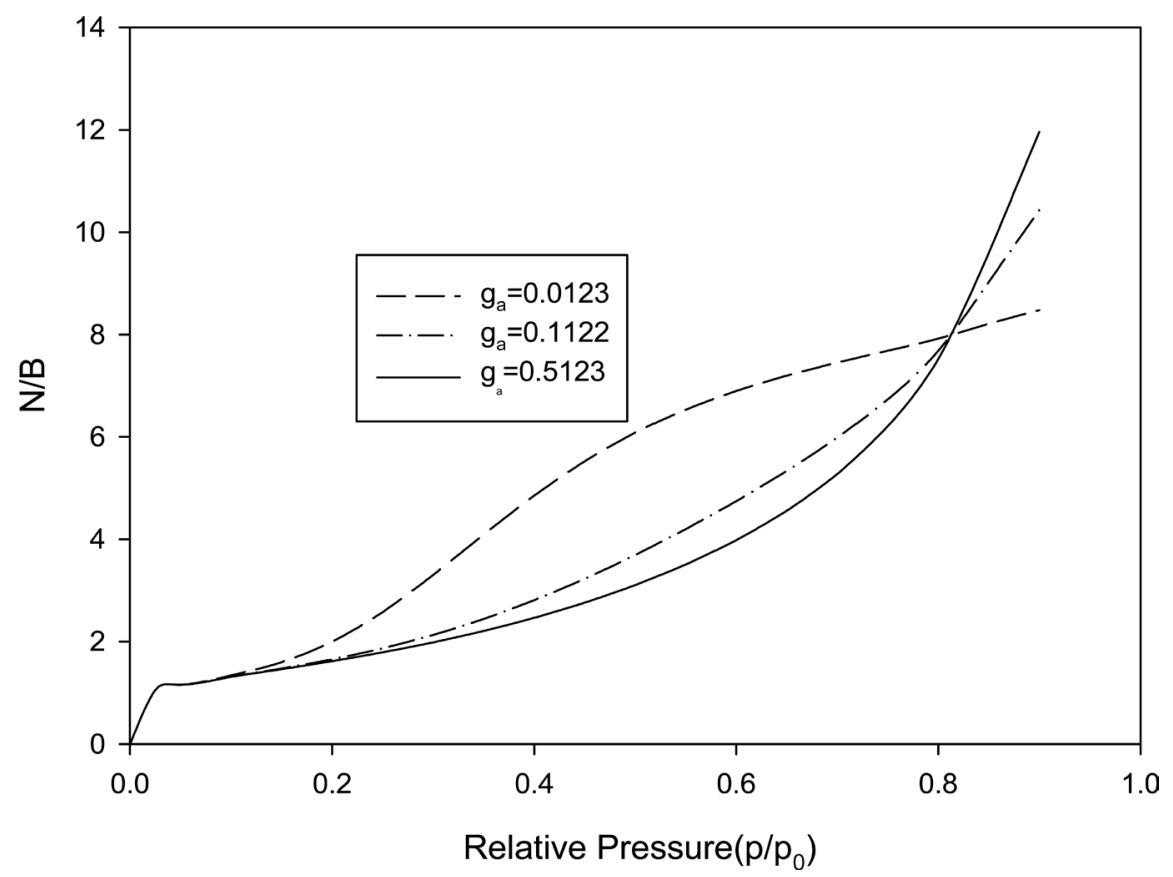

Figure 5. Theoretical adsorption isotherm curve, Equation (12) $\left(\mathrm{m}=1.7, \mathrm{n}=8, \beta_{\mathrm{a}}=0.009\right)$ with respect to $\mathrm{g}_{\mathrm{a}}$ values. 


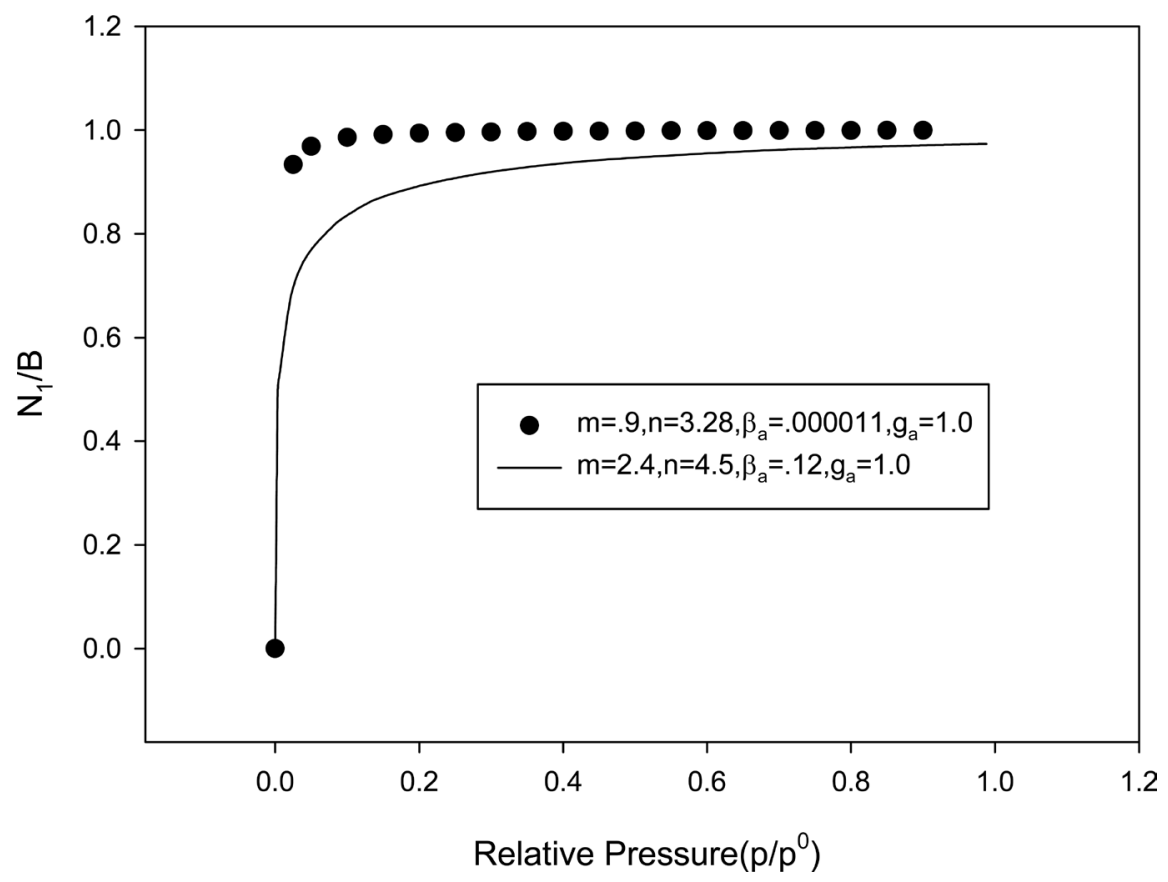

Figure 6. Theoretical adsorption isotherm of the first layer using Equation (10) including experimental constants of Figure 7 and Figure 8.

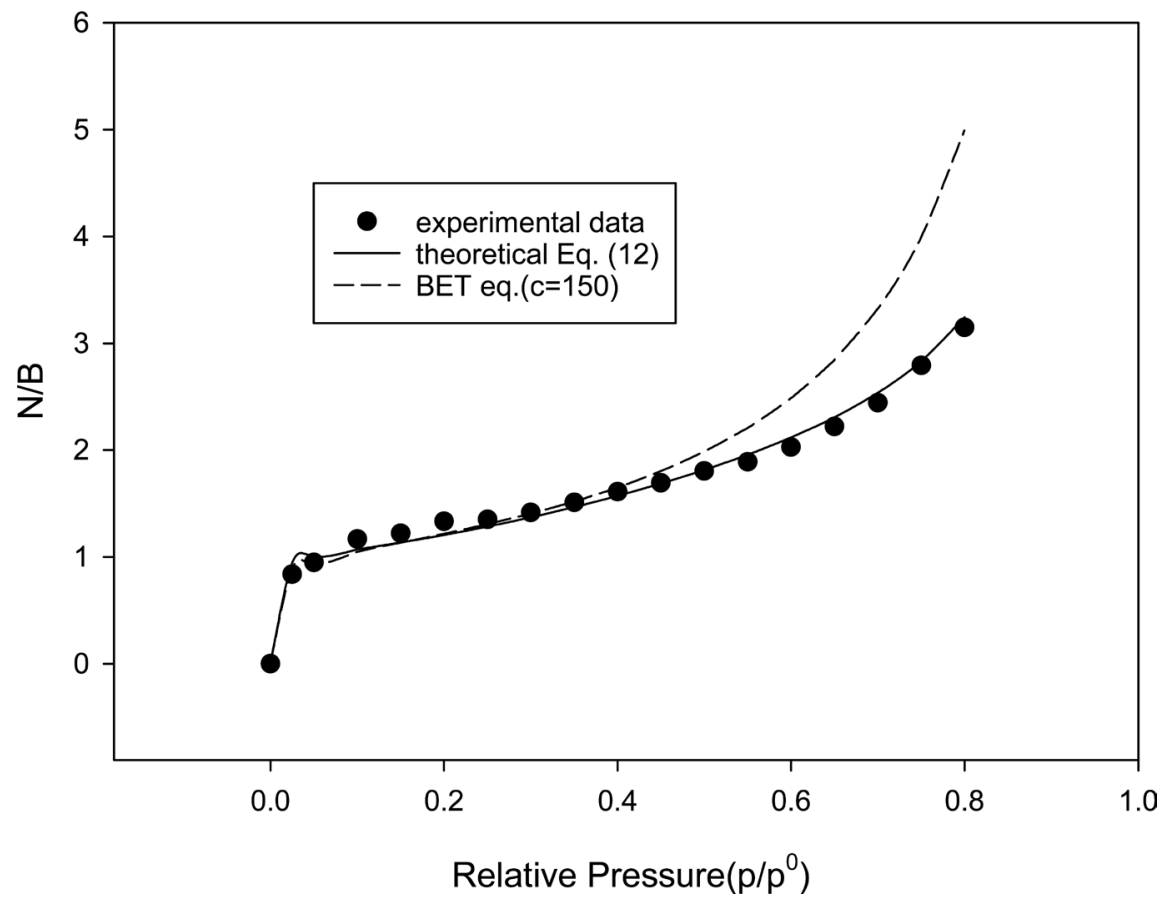

Figure 7. Theoretical adsorption isotherm, Equation $(12)\left(\mathrm{m}=0.9, \mathrm{n}=3.28, \beta_{\mathrm{a}}=0.000011, \mathrm{~g}_{\mathrm{a}}=1.0\right)$ with BET equation (c $=$ 150 ) and experimental nitrogen adsorption at $-196^{\circ}$ on non-porous samples of silica and aluminna [6].

experimental data are fitted to Equation (12) well. As we see in Figure 7 and Figure 8, BET isotherms there can't imitate the experimental data except for beginning.

Equation (13) can be used in the data fitting with $m=1.0$ and $g_{a}=1.0$. Its quality is poor.

What is the catalyst? As we see Figure 9, 1, 2, 3, 4, 5, 6 and 7 molecules can function as the catalyst. That is, the molecules of the surface adsorption layer can't function like the catalyst. Because they use much 


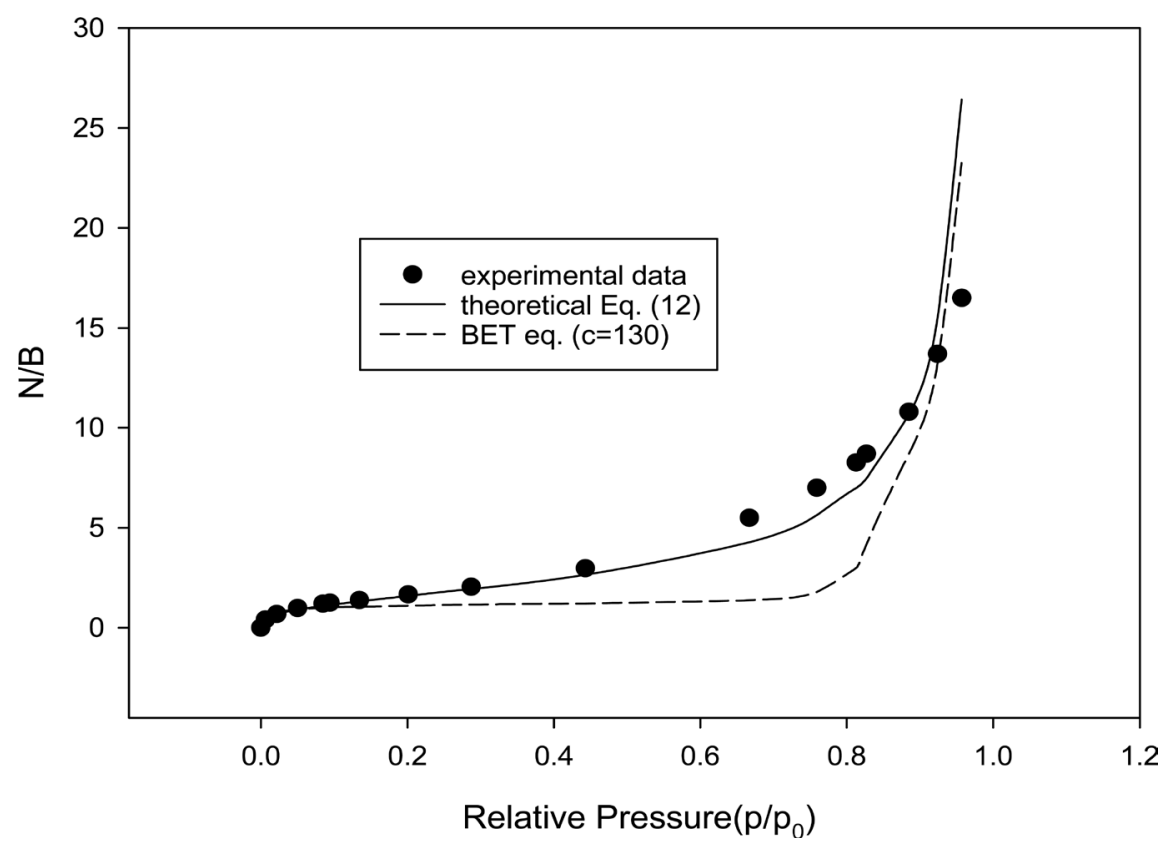

Figure 8. Theoretical adsorption isotherm curve, Equation $(12)\left(\mathrm{m}=2.4, \mathrm{n}=4.5, \beta_{\mathrm{a}}=0.12, \mathrm{~g}_{\mathrm{a}}=1.0\right)$ with BET equation (c $=$ 130) curve and experimental water adsorption at $25^{\circ}$ on cross-linked polystyrene sulfuric acid resin [7].

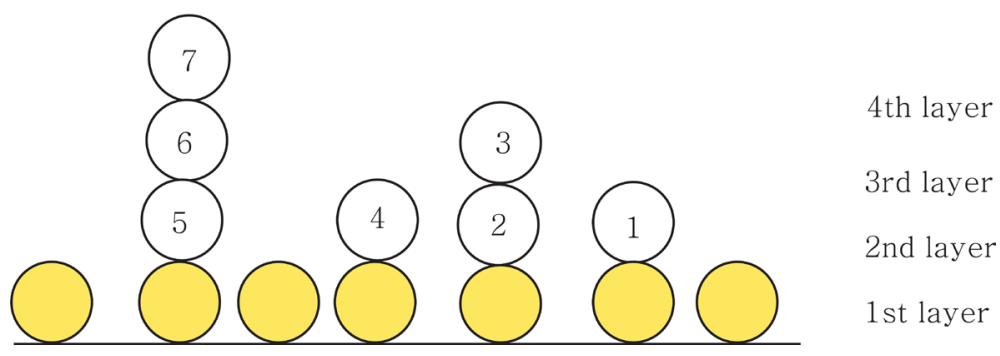

Figure 9. Real adsorption molecules to get the surface area (numbered rings).

energy in order to hold the surface. So they are not active. The molecules which lie on the surface layer adsorption molecules which hold the surface, can function as the real catalyst. Therefore in Equation (12) $\frac{1}{\beta_{a}+a}\left\{\left(\frac{z^{\frac{2}{m}}-z^{\frac{n}{m}}}{\left(1-z^{\frac{1}{m}}\right)^{2}}+\frac{n-1}{g_{a}} z^{\frac{n}{m}}\right)\right\}$ part must work in the adsorption reaction. Hence the real adsorption isotherm equation becomes

$$
\frac{N_{\text {real }}}{B}=\frac{1}{\beta_{a}+a}\left\{\left(\frac{z^{\frac{2}{m}}-z^{\frac{n}{m}}}{\left(1-z^{\frac{1}{m}}\right)^{2}}+\frac{n-1}{g_{a}} z^{\frac{n}{m}}\right)\right\}
$$

If this equation is integrated from the inflection point $\left(z_{i}\right)$ to each relative pressure $\left(p / p_{0}\right)$, we get the mono-area capacity $\left(S_{m}\right)$ with respect to the relative pressure $\left(p / p_{0}\right)$. Table 1 and Table 2 include those. Then 
Table 1. $S_{m}$ (mono-area capacity) and $S$ (surface area) for Figure 7.

\begin{tabular}{|c|c|c|}
\hline $\begin{array}{l}p / p_{0} \text { integration interval Inflection } \\
\text { point }\left(z_{i}=0.573\right)\end{array}$ & $\begin{array}{c}S_{m} \text { for Figure } 7(m=0.9, n=3.28, \\
\left.\beta_{a}=0.000011, g_{a}=1.0\right) \quad S_{m}=\int_{z_{i}}^{z_{m}} \frac{N_{\text {real }}}{B} \mathrm{~d} z\end{array}$ & $\begin{array}{c}S\left(\mathrm{~m}^{2} / \mathrm{g}\right) \text { for Figure } 7=\text { Surface } \\
\text { Area of Catalyst, Nitrogen }\end{array}$ \\
\hline $0.573-0.6$ & 0.000042 & 0.2927 \\
\hline $0.573-0.7$ & 0.000199 & 1.3869 \\
\hline $0.573-0.8$ & 0.000355 & 2.4741 \\
\hline $0.573-0.9$ & 0.000512 & 3.5683 \\
\hline
\end{tabular}

Table 2. $S_{m}$ (mono-area capacity) and $S$ (surface area) for Figure 8.

\begin{tabular}{ccc}
\hline $\begin{array}{c}\mathrm{p} / \mathrm{p}_{0} \text { integration interval Inflection } \\
\text { point }\left(z_{i}=0.26\right)\end{array}$ & $S_{m}$ for Figure $8\left(m=2.4, n=4.5, \quad \beta_{a}=0.12, g_{a}=1.0\right)$ & $\begin{array}{c}S\left(\mathrm{~m}^{2} / \mathrm{g}\right) \\
\text { Area of Catalyst, Water }\end{array}$ \\
\hline $0.26-0.3$ & $S_{m}=\int_{z_{i}}^{2_{m}} \frac{N_{r e a l}}{B} \mathrm{~d} z .0 .00175$ & 5.5968 \\
$0.26-0.4$ & 0.00615 & 19.6690 \\
$0.26-0.5$ & 0.01050 & 33.5812 \\
$0.26-0.6$ & 0.01490 & 47.6533 \\
$0.26-0.7$ & 0.01930 & 61.6665 \\
$0.26-0.8$ & 0.02372 & 76.0261 \\
$0.26-0.9$ & 0.02810 & 89.8698 \\
\hline
\end{tabular}

the surface area for the catalyst obtained from the equation becomes $S\left(\mathrm{~m}^{2} / \mathrm{g}\right)=\left(S_{m} / M\right) \cdot N \cdot A_{m} \times 10^{-20}$ with $A_{m}=16.2 \AA^{2}$ per number for nitrogen and $A_{m}=0.59 \times 16.2 \AA^{2}$ per number for water (Table 2.12) [6] by substituting $x_{m}$. in the equation of (2.60) of the reference [6] by $S_{m}=\int_{z_{i}}^{z_{m}} \frac{N_{\text {real }}}{B} \mathrm{~d} z$ in which $z_{i}=$ inflection point and $z_{m}=$ optional $z$ value after the inflection point. In the above equation $M$ is the molecular weight (g) per g-mole and $N$ Avogadro number per g-mole.

Then the inflection points are obtained by Secant method [8] through the program showed in Appendix 1. Specific surface areas are changed according to the relative pressures. These are showed in Table 1 and Table 2 precisely. The integrations with respect to $\mathrm{z}$ values give the total adsorption site numbers of the adsorbate. Before the inflection point the specific surface area of the adsorbent is not counted as a catalyst since it makes the strong surface film [9]. The adsorption rate increases consistently after the inflection point. The values of $n$ of Figure 7 and Figure 8 match the range of the reference of BET [10]. The completion of $N_{1}$ goes with the completion of $N$ to the end as we see in Figure 6 and its equation. But from the inflection point the plugging of $N_{1} / B$ may begin characteristically.

We have felt intimately that the adsorption molecules of more than $2^{\text {nd }}$ layers lie down the first layer molecules softly since the small quantity of adsorption molecules control the drawing and the surface area of numerical number.

Our study have realized the saying that "After considerable work on the theory, Hill (1946) formed the opinion that any future improvement on it must be in the form of refinement rather than a modification on the basic theory” [11] [12].

\section{Conclusion}

The total adsorption rate equations closely related with the past references are derived correctly and the figures according to four constants $\left(m, n, \beta_{a}, g_{a}\right)$ are also considered to describe BET-like figures (type II) well. The quantization constants are useful in deriving the adsorption equations. In order to calculate the surface area of 
the catalysts, the total adsorption equations excluding the first layer adsorption equation and their inflection points obtained are used appropriately. They fit the experimental data well.

\section{Acknowledgements}

The author thanks for the encouragements of Yongduk Kim, an emeritus professor of Sogang University.

\section{References}

[1] Reif, F. (1985) Fundamentals of Statistical and Thermal Physics. McGraw-Hill Inc Ed. 1965, Chapter 1, 10-46.

[2] Kim, D., Lee, Y. and Kim, T.-W. (2010) Article Title. New Physics: Sae Mulli (The Korean Physical Society), 60, 729740.

[3] Mathews, J.H. and Howell, R.W. (2001) Complex Analysis for Mathematics and Engineering. 4th Edition, Jones and Bartlett Publishers, Inc., Section 2-3, 62-69.

[4] Kim, D. (2000) Statistical Condensation Adsorption Isotherms of Gas Molecules Adsorbed on Porous Adsorbents, Surface Monolayer Adsorption Isotherms and Hysteresis Phenomena. Korean Journal of Chemical Engineering, 17, 600-612. http://dx.doi.org/10.1007/BF02707174

[5] Kim, D. and Choi, Y.S. (2011) Brightness of Lena's Image for Various $\gamma$ Values by Using Differentials of the Geometric Mean Heat Capacity Equations at Constant Volume. New Physics: Sae Mulli (The Korean Physical Society), 61, 248-255. http://dx.doi.org/10.3938/NPSM.61.248

[6] Gregg, S.J. and Sing, K.S.W. (1969) Adorption, Surface Area and Porosity. Academic Press Inc. (Ltd.), Chapter 2, 53, 77.

[7] Sundheim, B.R., Waxman, M.H. and Gregor, H.P. (1953) J Phys Chem., 57, 974-978.

[8] Ji, Y., Kim, H. and Heo, J. (2002) C-Roguhyouhan Numerical Analysis (Korean Language). Nopi-gipi Publishing Company, 24-27.

[9] Jura, G. and Harkins, W.D. (1944) Surfaces of Solids. XI. Determination of the Decrease ( $\pi$ ) of Free Surface Energy of a Solid by an Adsorbed Film. Journal of the American Chemical Society, 67, 1356-1362. http://dx.doi.org/10.1021/ja01236a046

[10] Brunauer, S., Emmett, P.H. and Teller, E. (1938) J. of Amer. Chem. Soc., 90, 309-319.

[11] Caurie, M. (The Year after 1979) J. Food Sci.

[12] Caurie, M. (The year after 1979) J. Food Sci., 63. 


\section{Appendix 1}

/*--Finding zero by Secant method [8] to get inflection point from Eq. (12). with c++ and c languages */

\#include $<$ iostream $>$

using namespace std;

\#include $<$ stdio.h $>$

\#include $<$ math.h $>$

\#include $<$ conio.h $>$

float eval_f(float $\mathrm{x})$; //evaluation of $\mathrm{f}(\mathrm{x})$

float fprime(float x1,float x2); //diff. of f(a)

void main()

$\{$ float x1,x2,xn,e;

int $\mathrm{i}=0$;

printf(“"nType initial point: “);

scanf(“\%f”, \&x1);

printf(“'nType second point: “);

scanf(“\%f”,\&x2);

printf(“'nType acceptable error interval in y: “);

scanf(“\%f”, \&e);

$\mathrm{i}=0$;

do

$\{\mathrm{xn}=\mathrm{x} 1-\mathrm{eva} ; \mathrm{f}(\mathrm{x} 1) / \mathrm{fprime}(\mathrm{x} 1, \mathrm{x} 2)$;

$\mathrm{x} 1=\mathrm{x} 2$;

$\mathrm{x} 2=\mathrm{xn}$;

printf(“ $\mid$ n\%3dth Iteration Root :\%f < with error \%f $>\mid \mathrm{n}$ ”, $++\mathrm{i}, \mathrm{xn}, \mathrm{fabs}($ eval_f(xn)));

getch();

\} while(fabs(eval_f(xn))>e );

\}

float eval_f(float $\mathrm{x})$

$\{$ float $\mathrm{b}$;

//cout $<<$ " $\mathrm{x}=$ " $<<\mathrm{x}<<$ endl;

double bc;

double bb,bb1,bb2,cc,dd,dd1,dd2,dd3,ee,ee1,ee2,ff,ff1,ff2;

double aa,aa1,aa2,ba1,ba2,ba3,ccc1,ccc2,cc1,cc2,cc3,cc4,cc11,cc12;

const double $\mathrm{b} 1=.000091, \mathrm{ga}=.983$, $\mathrm{an}=10.0, \mathrm{am}=.64$;

double ad21,ad22,ad23;

$\mathrm{bb}=\operatorname{pow}(\mathrm{x},(1 . / \mathrm{am}))-\operatorname{pow}(\mathrm{x},(\mathrm{an} / \mathrm{am}))$;

bb1=(1./am)*pow(x,(1./am)-1.))-(an/am)*pow(x,(an/am-1.));

bb2=(1./am)*(1./am-1.)*pow(x,(1./am)-2.))-(an/am)*(an/am-1.)*pow(x,(an/am-2.));

ccc1=-(1./am)*pow(x,,1./am-1.);

cсc2=-(1./am)*(1./am-1.)*pow(x,1./am-2.);

cc1=pow((1.-pow(x,1./am)),-1.);

cc2=pow((1.-pow(x,1./am)),-2.);

cс3=pow((1.-pow(x,1./am)),-3.);

cc4=pow((1.-pow(x,1./am)),-4.);

$\mathrm{dd}=\operatorname{pow}(\mathrm{x},(\mathrm{an} / \mathrm{am})) / \mathrm{ga}$;

dd1 $=($ an/am $) * \operatorname{pow}(x,((a n / a m)-1).) / g a ;$

$\mathrm{dd} 2=(\mathrm{an} / \mathrm{am}) *(\mathrm{an} / \mathrm{am}-1). * \operatorname{pow}(\mathrm{x},((\mathrm{an} / \mathrm{am})-2).) / \mathrm{ga}$; 
ee=pow(x,2./am)-pow(x,an/am);

ee1=(2./am)*pow(x,(2./am)-1.)-(an/am)*pow(x,(an/am)-1.);

ee2=((2./am)*(2./am-1.)*pow(x,(2./am)-2.)-(an/am)*(an/am-1.)*pow(x,(an/am-2.);

$\mathrm{ff}=((\mathrm{an}-1.) / \mathrm{ga}) * \operatorname{pow}(\mathrm{x}, \mathrm{an} / \mathrm{am})$;

ff1 $=(($ an-1.)/ga $) *(a n / a m) * \operatorname{pow}(x,(a n / a m)-1$.$) ;$

ff2=((an-1.)/ga)*(an/am)*(an/am-1.)*pow(x,(an/am)-2.);

$\mathrm{aa}=\mathrm{bb} * \mathrm{cc} 1+\mathrm{dd}$;

aa $1=\mathrm{bb} 1 * \mathrm{cc} 1+(-1) * \mathrm{cc} 2 * \mathrm{ccc} 1 * \mathrm{bb}+\mathrm{dd} 1$

aa2 $=\mathrm{bb} 2 * \mathrm{cc} 1+(-1) * \mathrm{cc} 2 * \mathrm{ccc} 1 * \mathrm{bb} 1+(-1) *(-2) * \mathrm{cc} 3 * \mathrm{ccc} 1{ }^{*} \mathrm{ccc} 1 * \mathrm{bb}+(-1) * \mathrm{ccc} 2 * \mathrm{cc} 2 * \mathrm{bb}+(-1) * \mathrm{bb} 1 * \mathrm{cc} 2 * \mathrm{ccc} 1+\mathrm{dd} 2$;

ba1=pow((b1+aa),-1);

ba2=pow((b1+aa),-2);

ba3=pow((b1+aa),-3);

ad21=aa2*ba1+(-1)*ba2*aa1*aa1+(-1)*(-2)*ba3*aa1*aa1*aa+(-1)*aa2*ba2*aa+(-1)*aa1*aa1*ba2;

ad22 $=$ ee $2 *$ cc $2 *$ ba $1+(-2) *$ cc $3 *$ ccc $1 * e e 1 *$ ba $1+(-1) *$ ba $2 * a a 1 * e e * c 2+(-2) *(-3) * c c 4 * c c c 1 * c c c 1 * e e * b a 1+(-2) *$ ccc $2 *$ cc2*ee*ba1+(-2)*ee1*cc3*ccc1*ba1+(-2)*(-1)*ba2*aa1*cc3*ccc1*ee+(-1)*(-2)*ba3*aa1*aa1*ee*cc2+(-1)* aa2*ba2*ee*cc $2+(-1) * e e 1 *$ ba $2 *$ aa $1 *$ cc $2+(-1) *(-2) *$ cc $3 *$ ccc $1 *$ ba $2 *$ aa $1 * e e 1 ;$

ad23=ff $*$ ba $1+(-1) * \mathrm{ba} 2 * \mathrm{aa} 1 * \mathrm{ff} 1+(-1) *(-2) * \mathrm{ba} 3 * \mathrm{aa} 1 * \mathrm{aa} 1 * \mathrm{ff}+(-1) * \mathrm{aa} 2 * \mathrm{ff} * \mathrm{ba} 2+(-1) * \mathrm{ff} 1 * \mathrm{ba} 2 * \mathrm{aa} 1 ;$

$\mathrm{b}=\mathrm{ad} 21+\mathrm{ad} 22+\mathrm{ad} 23$;

return(b);

\}

float fprime (float x1,float x2)

$\{$ float b6;

b6=(eval_f(x2)-eval_f(x1))/(x2-x1);

return(b6);

\} 